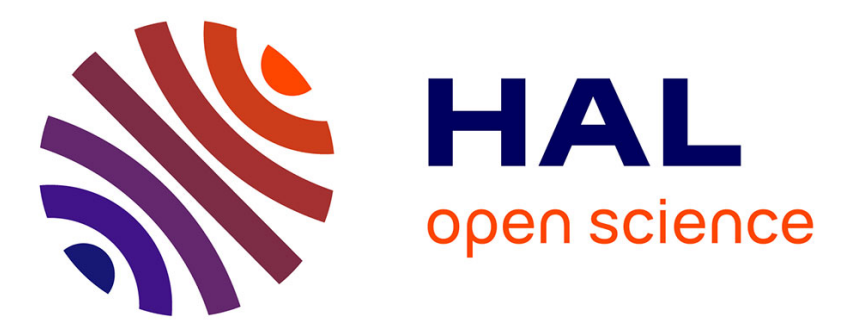

\title{
Full waveform inversion of seismic waves reflected in a stratified porous medium
}

\author{
Louis de Barros, Michel Dietrich, Bernard Valette
}

\section{To cite this version:}

Louis de Barros, Michel Dietrich, Bernard Valette. Full waveform inversion of seismic waves reflected in a stratified porous medium. Geophysical Journal International, 2010, 182, pp.1543-1556. 10.1111/J.1365-246X.2010.04696.X . insu-00565020

\section{HAL Id: insu-00565020 \\ https://hal-insu.archives-ouvertes.fr/insu-00565020}

Submitted on 12 Mar 2021

HAL is a multi-disciplinary open access archive for the deposit and dissemination of scientific research documents, whether they are published or not. The documents may come from teaching and research institutions in France or abroad, or from public or private research centers.
L'archive ouverte pluridisciplinaire $\mathbf{H A L}$, est destinée au dépôt et à la diffusion de documents scientifiques de niveau recherche, publiés ou non, émanant des établissements d'enseignement et de recherche français ou étrangers, des laboratoires publics ou privés. 


\title{
Full waveform inversion of seismic waves reflected in a stratified porous medium
}

\author{
Louis De Barros, ${ }^{1,2}$ Michel Dietrich ${ }^{1,3}$ and Bernard Valette ${ }^{4}$ \\ ${ }^{1}$ Laboratoire de Géophysique Interne et Tectonophysique, CNRS, Université Joseph Fourier, BP 53, 38041 Grenoble Cedex 9, France \\ ${ }^{2}$ School of Geological Sciences, University College Dublin, Belfield, Dublin 4, Ireland. E-mail: louis.debarros@ucd.ie \\ ${ }^{3}$ Geophysics Department, IFP, 92852 Rueil Malmaison, France \\ ${ }^{4}$ Laboratoire de Géophysique Interne et Tectonophysique, IRD, CNRS, Université de Savoie, 73376 Le Bourget du Lac Cedex, France
}

Accepted 2010 June 8. Received 2010 June 8; in original form 2010 February 18

\begin{abstract}
SUMMAR Y
In reservoir geophysics applications, seismic imaging techniques are expected to provide as much information as possible on fluid-filled reservoir rocks. Since seismograms are, to some degree, sensitive to the mechanical parameters and fluid properties of porous media, inversion methods can be devised to directly estimate these quantities from the waveforms obtained in seismic reflection experiments. An inversion algorithm that uses a generalized least-squares, quasi-Newton approach is described to determine the porosity, permeability, interstitial fluid properties and mechanical parameters of porous media. The proposed algorithm proceeds by iteratively minimizing a misfit function between observed data and synthetic wavefields computed with the Biot theory. Simple models consisting of plane-layered, fluid-saturated and poro-elastic media are considered to demonstrate the concept and evaluate the performance of such a full waveform inversion scheme. Numerical experiments show that, when applied to synthetic data, the inversion procedure can accurately reconstruct the vertical distribution of a single model parameter, if all other parameters are perfectly known. However, the coupling between some of the model parameters does not permit the reconstruction of several model parameters at the same time. To get around this problem, we consider composite parameters defined from the original model properties and from a priori information, such as the fluid saturation rate or the lithology, to reduce the number of unknowns. Another possibility is to apply this inversion algorithm to time-lapse surveys carried out for fluid substitution problems, such as $\mathrm{CO}_{2}$ injection, since in this case only a few parameters may vary as a function of time. We define a two-step differential inversion approach which allows us to reconstruct the fluid saturation rate in reservoir layers, even though the medium properties are poorly known.
\end{abstract}

Key words: Inverse theory; Permeability and porosity; Controlled source seismology; Computational seismology.

\section{INTRODUCTION}

The quantitative imaging of the subsurface is a major challenge in geophysics. In oil and gas exploration and production, deep aquifer management and other applications such as the underground storage of $\mathrm{CO}_{2}$, seismic imaging techniques are implemented to provide as much information as possible on fluid-filled reservoir rocks. The Biot theory (Biot 1956) and its extensions (e.g. Auriault et al. 1985; Pride et al. 1992; Johnson et al. 1994) provide a convenient framework to connect the various parameters characterizing a porous medium to the wave properties, namely, their amplitudes, velocities and frequency content. The poroelastic model involves more parameters than the elastodynamic theory, but on the other hand, the wave attenuation and dispersion characteristics at the macroscopic scale are determined by the medium intrinsic properties without having to resort to empirical relationships. Attenuation mechanisms at microscopic and mesoscopic scales, which are not considered in the original Biot theory, can be introduced into alternative poroelastic theories (see e.g. Pride et al. 2004). The inverse problem, that is, the retrieval of poroelastic parameters from the seismic waveforms is much more challenging. Porosity, permeability and fluid saturation are the most important parameters for reservoir engineers. Being related to seismic wave attenuation, permeability appears as not only the most difficult parameter to estimate but also the one which would have the greatest benefits to the characterization of porous formations, notably in the oil industry (Pride et al. 2003).

The estimation of poroelastic properties of reservoir rocks is still in its infancy. One way to solve this problem is to first determine the seismic wave velocities by using an elastic representation 
of the medium. In a second stage, the velocities are interpreted in terms of poroelastic parameters by using deterministic rock physics transforms (Domenico 1984; Berryman et al. 2002). However, this approach does not resolve the ambiguities between the parameters. A second class of methods is based on stochastic rock physics modelling, such as Monte-Carlo methods (Mosegaard \& Tarantola 2002). This approach is then combined with a Bayesian description of the porous medium to evaluate the probability distribution of the parameters (Mukerji et al. 2001). For example, Bachrach (2006) inverts for the seismic impedances to determine the porosity and fluid saturation. Spikes et al. (2007) estimate the clay content, fluid saturation and porosity from the seismic waveforms. Larsen et al. (2006) use an Amplitude Versus Offset analysis to infer the properties of porous media. Bosch (2004) introduces a lithological least-squares inversion technique by combining seismic data and petrophysics for porosity prediction. However, this method does not make full use of the seismograms.

The aim of this paper is to investigate the feasibility of a full waveform inversion (FWI) of the seismic response of plane-layered, fluid-saturated media to estimate the poroelastic parameters of each layer using simple numerical experiments. To our knowledge, the use of the Biot theory in 'direct' FWI has been seldom addressed. Sensitivity kernels have been derived by De Barros \& Dietrich (2008) for 1-D media and by Morency et al. (2009) for 3-D media. Historically, most of the FWI methods (Lailly 1983; Tarantola 1984; Mora 1987) have been implemented under the acoustic approximation, for 2-D model reconstruction (e.g. Gauthier et al. 1986; Pratt et al. 1998) or 3-D structures (for instance, Ben-Hadj-Ali et al. 2008; Sirgue et al. 2008). Applications to real data are even more recent (Pratt \& Shipp 1999; Hicks \& Pratt 2001; Operto et al. 2006). The elastic case is more challenging, as the coupling between $P$ and $S$ waves leads to ill-conditioned problems. Since the early works of Mora (1987) and Kormendi \& Dietrich (1991), the elastic problem has been addressed several times over the last years with methodological developments (Gélis et al. 2007; Choi et al. 2008; Brossier et al. 2009).

In the poroelastic case, eight model parameters enter the medium description, compared with only one or two in the acoustic case, and three in the elastic case if wave attenuation is not taken into account. The advantages of using a poroelastic theory in FWI are (1) to directly relate seismic wave characteristics to porous media properties; (2) to use information that cannot be described by viscoelasticity or elasticity with the Gassmann (1951) formula and (3) to open the possibility to use fluid displacement and force to determine permeability and fluid properties. The assumption of plane-layered media is admittedly too simple to correctly describe the structural features of geological media, but it is nevertheless useful to explore the feasibility of an inversion process accounting for the rheology of porous media. As shown by De Barros \& Dietrich (2008), the perturbation of different model parameters may lead to similar seismic responses. This observation stresses the fact that the major issue to solve is to find a viable strategy to efficiently reconstruct the most relevant parameters of poroelastic media. Nowadays, exploration seismology more and more relies on 4-D monitoring, that is, the observation of the space-time variations of the Earth response. For example, underground $\mathrm{CO}_{2}$ storage operations use time-lapse surveys to assess the spatial extent of the $\mathrm{CO}_{2}$ plume and to detect and locate possible $\mathrm{CO}_{2}$ leakage. In this case, the most important parameters required to describe the time variations of the medium properties are those related to the injected fluid and to the fluid in place.
We first outline in Section 2 the poroelastic theory used to solve the forward modelling of seismic wave propagation in poroelastic media. This theory is implemented for layered media with the generalized reflection and transmission matrix method of Kennett (1983). This computation method is checked with the semi-analytical solution of Philippacopoulos (1997) for a homogeneous half-space. In Section 3, we briefly present the generalized least-squares inversion procedure (Tarantola \& Valette 1982) and the quasi-Newton algorithm that we have implemented following the formalism of Tarantola (1987). Section 4 of the article is dedicated to accuracy and stability checks of the inversion algorithm with synthetic data. Section 5 discusses and quantifies the coupling between model parameters. Finally, Section 6 deals with strategies developed to circumvent this coupling problem. We introduce composite model parameters such as the fluid saturation rate and lithology, and study their use to monitor time variations of the medium properties via a two-step differential inversion approach.

\section{WAVE PROPAGATION IN STRATIFIED POROUS MEDIA}

\subsection{Governing equations}

Assuming a $\mathrm{e}^{-\mathrm{i} \omega \mathrm{t}}$ dependence, Pride et al. (1992) rewrote Biot's (1956) equations of poroelasticity in the form

$$
\begin{aligned}
& {\left[\left(K_{U}+G / 3\right) \nabla \nabla+\left(G \nabla^{2}+\omega^{2} \rho\right) \boldsymbol{I}\right] \cdot \mathbf{u}} \\
& \quad+\left[C \nabla \nabla+\omega^{2} \rho_{f} \boldsymbol{I}\right] \cdot \mathbf{w}=0 \\
& {\left[C \nabla \nabla+\omega^{2} \rho_{f} \boldsymbol{I}\right] \cdot \mathbf{u}+\left[M \nabla \nabla+\omega^{2} \tilde{\rho} \boldsymbol{I}\right] \cdot \mathbf{w}=0,}
\end{aligned}
$$

where $\mathbf{u}$ and $\mathbf{w}$, respectively, denote the average solid displacement and the relative fluid-to-solid displacement, $\omega$ is the angular frequency, $\boldsymbol{I}$ the identity tensor, $\nabla \nabla$ the gradient of the divergence operator and $\nabla^{2}$ the Laplacian operator. The other quantities appearing in eqs (1) are medium properties. The bulk density of the porous medium $\rho$ is related to the fluid density $\rho_{f}$, solid density $\rho_{s}$ and porosity $\phi$

$\rho=(1-\phi) \rho_{s}+\phi \rho_{f}$.

$K_{U}$ is the undrained bulk modulus and $G$ is the shear modulus. $M$ (fluid storage coefficient) and $C$ (C-modulus) are mechanical parameters. In the quasi-static limit, at low frequencies, these parameters are real, frequency-independent and can be expressed in terms of the drained bulk modulus $K_{D}$, porosity $\phi$, mineral bulk modulus $K_{s}$ and fluid bulk modulus $K_{f}$ (Gassmann 1951):

$$
\begin{aligned}
K_{U} & =\frac{\phi K_{D}+\left[1-(1+\phi) \frac{K_{D}}{K_{s}}\right] K_{f}}{\phi(1+\Delta)}, \\
C & =\frac{\left[1-\frac{K_{D}}{K_{s}}\right] K_{f}}{\phi(1+\Delta)}, \quad M=\frac{K_{f}}{\phi(1+\Delta)} \\
\text { with } \Delta & =\frac{1-\phi}{\phi} \frac{K_{f}}{K_{s}}\left[1-\frac{K_{D}}{(1-\phi) K_{s}}\right] .
\end{aligned}
$$

It is also possible to link the frame properties $K_{D}$ and $G$ to the porosity and constitutive mineral properties (Korringa et al. 1979; Pride 2005):

$K_{D}=K_{s} \frac{1-\phi}{1+c_{s} \phi} \quad$ and $\quad G=G_{s} \frac{1-\phi}{1+3 c_{s} \phi / 2}$,

where $G_{s}$ is the shear modulus of the grains. The consolidation parameter $c_{s}$ appearing in these expressions is not necessarily the 
same for $K_{D}$ and $G$ (Korringa et al. 1979; Walton 1987). However, to minimize the number of model parameters, and following the recommendation of Pride (2005), we consider only a single consolidation parameter to describe the frame properties. Parameter $c_{s}$ typically varies between 2 and 20 in a consolidated medium, but can be much greater than 20 in an unconsolidated soil.

Finally, the wave attenuation is explained by a generalized Darcy's law, which uses a complex, frequency-dependent dynamic permeability $k(\omega)$ defined via the relationship (Johnson et al. 1994)

$\tilde{\rho}=\mathrm{i} \frac{\eta}{\omega k(\omega)} \quad$ with $\quad k(\omega)=k_{0} /\left[\sqrt{1-\mathrm{i} \frac{4}{n_{J}} \frac{\omega}{\omega_{c}}}-\mathrm{i} \frac{\omega}{\omega_{c}}\right]$.

In eq. (5), $\eta$ is the viscosity of the fluid and $k_{0}$ the hydraulic (dc) permeability. Parameter $n_{J}$ is considered constant and equal to 8 to simplify the equations. The relaxation frequency $\omega_{c}=\eta /\left(\rho_{f} F k_{0}\right)$, where $F$ is the electrical formation factor, separates the lowfrequency regime where viscous losses are dominant from the highfrequency regime where inertial effects prevail. We refer the reader to the work of Pride (2005) for more information on the parameters used in this study.

The solution of eq. (1) leads to classical fast $P$ and $S$ waves, and to additional slow $P$ waves (often called Biot waves) which can be seen at low frequency as a fluid pressure equilibration wave. Alternative poroelastic theories could have been considered, such as the one developed by Sahay (2008), which also considers slow $S$ waves. Although these theories are more advanced than the Biot theory, we will use the latter for sake of simplicity.

\subsection{Forward problem}

The model properties $\mathbf{m}$ consisting of the material parameters introduced in the previous section are non-linearly related to the seismic data $\mathbf{d}$ via an operator $f$, that is, $\mathbf{d}=f(\mathbf{m})$. The forward problem is solved in the frequency-wavenumber domain for horizontally layered media by using the generalized reflection and transmission method of Kennett (1983). The synthetic seismograms are finally transformed into the time-distance domain by using the 3-D axisymmetric discrete wavenumber integration technique of Bouchon (1981). This approach accurately treats multipathing and all mode conversions involving the fast and slow $P$ waves and the $S$ wave. It can also be used to obtain partial solutions to the full response, notably to remove the direct waves and surface waves from the computed wavefields. Both solid and fluid displacements are taken into account as the latter are partly responsible for the wave attenuation. This algorithm allows us to solve eq. (1) at all frequencies, that is, in the low- and high-frequency regimes, for forces consisting of stress discontinuities applied to a volume of porous rock and pressure gradients in the fluid. In this article, we exclusively concentrate on the low-frequency domain as seismic surveys are carried out in this regime.

This combination of techniques was first used by Garambois \& Dietrich (2002) to model the coupled seismic and electromagnetic wave propagation in stratified fluid-filled porous media. We have implemented a simplified version of this modelling scheme that retains only the seismic wave propagation. Similar methods have been developed by Haartsen \& Pride (1997) and by Pride et al. (2002).

To verify the accuracy of the modelling algorithm, we compare our numerical results with an analytical solution derived by Philippacopoulos $(1997,1998)$ in the frequency-wavenumber domain for a homogeneous medium bounded by a free surface. The time domain solution is obtained via an inverse Hankel transform followed by a Fourier transform. This solution has also been used by O'Brien (2010) to check a 3-D finite difference solution. The properties of the medium considered for the test are listed in Table 1 . The medium is excited by a vertical point force located $200 \mathrm{~m}$ below the free surface with a $40-\mathrm{Hz}$ Ricker wavelet time dependence. Relaxation frequency $\omega_{c}$ is equal to $16 \mathrm{kHz}$. Receivers are located at $100 \mathrm{~m}$ depth, regularly spaced horizontally at distances ranging from 0 to $200 \mathrm{~m}$ from the source. Fig. 1 shows that the agreement between our solution and that of Philippacopoulos (1998) is very good for vertical and horizontal displacements. The misfit values shown in Fig. 1 are defined as the rms value of the difference between the two solutions normalized by the rms value of the analytical solution. The consistency of our modelling algorithm has been further checked by using the reciprocity theorem, that is, by exchanging the source and the receiver in various configurations in a layered medium.

Our modelling algorithm is quite general and computes a full 3-D response, that is, it can handle the $P-S V$ and $S H$ wave propagation regimes. In this study, we concentrate on backscattered energy, that is, we consider reflected seismic waves as those recorded in seismic reflection experiments. We further assume that, whenever they exist, waves generated in the near surface (direct and head waves, surface and guided waves) are filtered out of the seismograms prior to the application of the inversion procedure. In the $P-S V$ case, the seismic response at the top of the layering can be computed in solid or fluid media to address land or marine applications.

The sensitivity of the seismic waveforms with respect to the model parameters is computed by using the poroelastic Fréchet derivatives recently derived by De Barros \& Dietrich (2008). These operators represent the first-order derivatives of the seismic displacements $\mathbf{d}$ with respect to the model properties $\mathbf{m}$. They can be readily and efficiently evaluated numerically because they are expressed as analytical formulae involving the Green's functions of the unperturbed medium.

In each layer, we consider the eight following quantities as model parameters: (1) the porosity $\phi$, (2) the mineral bulk modulus $K_{s}$, (3) the mineral density $\rho_{s}$, (4) the mineral shear modulus $G_{s}$, (5) the consolidation parameter $c_{s}$, (6) the fluid bulk modulus $K_{f}$, (7) the fluid density $\rho_{f}$ and (8) the permeability $k_{0}$. The fluid viscosity $\eta$ is one of the input parameters but it is not considered in the inversion tests as its sensitivity is very small and exactly similar to that of the permeability (De Barros \& Dietrich 2008). This parameter set allows us to distinguish the parameters characterizing the solid phase from those describing the fluid phase. Our parametrization differs from that used by Morency et al. (2009), as different parameter sets can be considered. Our inversion code is capable of inverting one model parameter at a time or several model parameters simultaneously.

Table 1. Model parameters of the homogeneous half-space model used for the algorithm check.

\begin{tabular}{ccccccccc}
\hline$\phi()$ & $k_{0}\left(\mathrm{~m}^{2}\right)$ & $\rho_{f}\left(\mathrm{~kg} \mathrm{~m}^{-3}\right)$ & $\rho_{s}\left(\mathrm{~kg} \mathrm{~m}^{-3}\right)$ & $K_{s}(\mathrm{GPa})$ & $G(\mathrm{GPa})$ & $K_{f}(\mathrm{GPa})$ & $K_{D}(\mathrm{GPa})$ & $\eta(\mathrm{Pa} \mathrm{s})$ \\
\hline 0.30 & $10^{-11}$ & 1000 & 2600 & 10 & 3.5 & 2.0 & 5.8333 & 0.001 \\
\hline
\end{tabular}



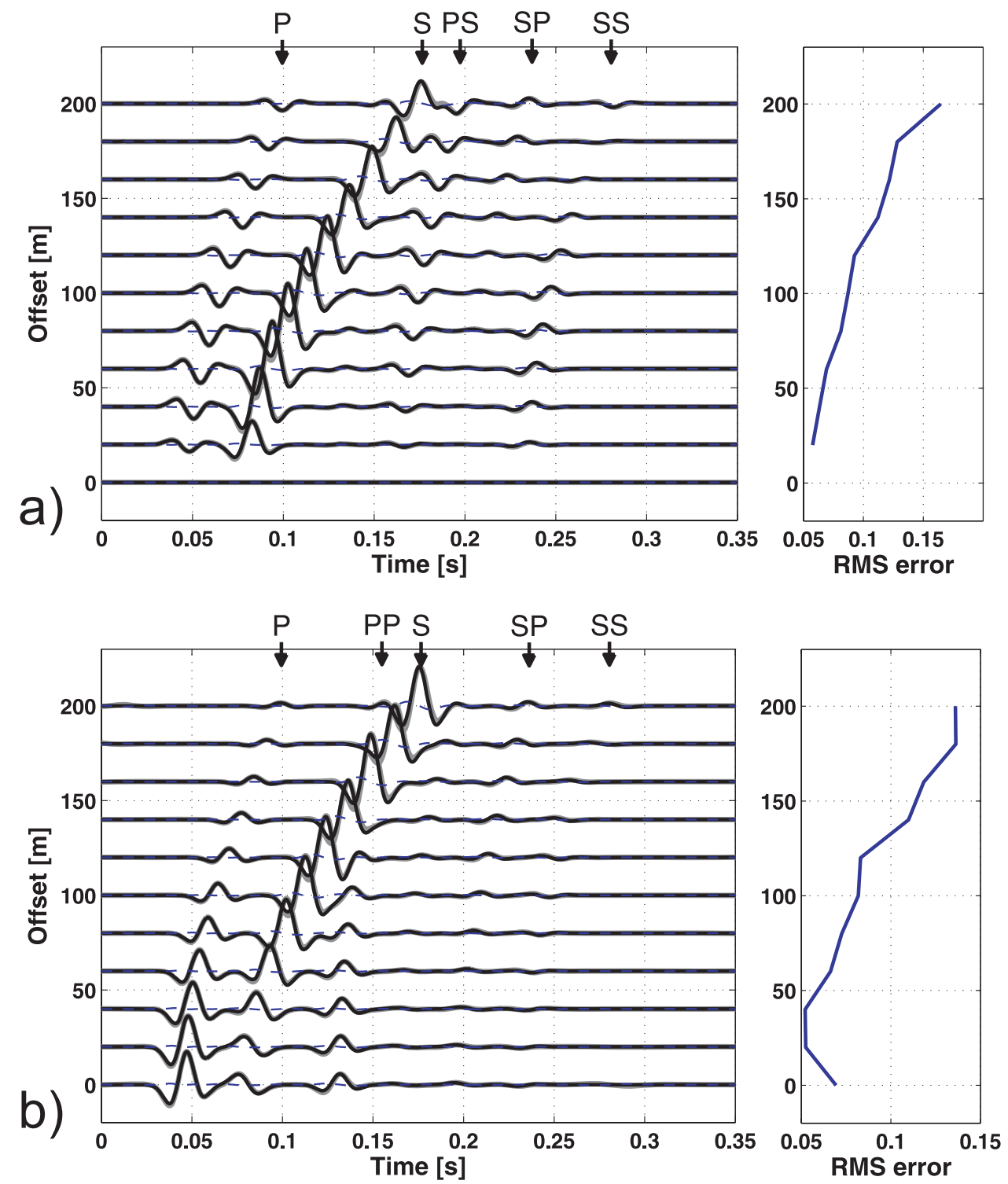

Figure 1. Left-hand panels: comparison of seismograms computed with our approach (solid black line) with the analytical solution of Philippacopoulos (1998) (thick grey line), and their differences (thin dashed line). Right-hand panels: Misfit between the two solutions. The comparisons are made for the (a) horizontal and (b) vertical solid displacements in a homogeneous half-space excited by a vertical point force. P and S denote the direct waves whereas PP, PS, SP and SS refer to the reflected and converted waves at the free surface.

\section{QUASI-NEWTON ALGORITHM}

The theoretical background of non-linear inversion of seismic waveforms has been presented by many authors, notably by Tarantola \& Valette (1982), Tarantola (1984, 1987) and Mora (1987). The inverse problem is iteratively solved by using a generalized least-squares formalism. The aim of the least-squares inversion is to infer an optimum model $\mathbf{m}_{\text {opt }}$ whose seismic response best fits the observed data $\mathbf{d}_{\mathrm{obs}}$, and which remains at the same time close to an a priori model $\mathbf{m}_{\text {prior }}$.

This optimum model corresponds to the minimum of a misfit (or cost) function $S(\mathbf{m})$ at iteration $n$ which is computed by a sampleby-sample comparison of the observed data $\mathbf{d}_{\text {obs }}$ with the theoretical seismograms $\mathbf{d}=f(\mathbf{m})$, and by an additional term which describes the deviations of the current model $\mathbf{m}$ with respect to the a priori model $\mathbf{m}_{\text {prior }}$, that is, (Tarantola \& Valette 1982; Tarantola 1987)

$S(\mathbf{m})=1 / 2\left(\left\|\mathbf{d}-\mathbf{d}_{\text {obs }}\right\|_{D}^{2}+\left\|\mathbf{m}-\mathbf{m}_{\text {prior }}\right\|_{M}^{2}\right)$.
The norms $\|.\|_{D}^{2}$ and $\|.\|_{M}^{2}$ are weighted $L_{2}$ norms, respectively, defined by

$$
\|\mathbf{d}\|_{D}^{2}=\mathbf{d}^{T} \boldsymbol{C}_{\boldsymbol{D}}{ }^{-1} \mathbf{d},
$$$$
\|\mathbf{m}\|_{M}^{2}=\mathbf{m}^{T} \boldsymbol{C}_{M}{ }^{-1} \mathbf{m},
$$

where $\boldsymbol{C}_{\boldsymbol{D}}$ and $\boldsymbol{C}_{\boldsymbol{M}}$ are covariance matrices.

The easiest way to include prior knowledge on the medium properties is through the model covariance matrix (e.g. Gouveia \& Scales 1998). Here we just want to limit the domain space without any further information. Thus, the model covariance matrix $\boldsymbol{C}_{\boldsymbol{M}}$ is assumed to be diagonal, which means that each model sample is considered independent from its neighbours. In practice, for each parameter in a given layer, we assign a standard deviation equal to a given percentage of its prior value. The diagonal terms of the matrix are thus proportional to the square of the prior values of the parameters for the different layers. This ensures that the influence of the different model parameters is of the same order of magnitude in 
the inversion, and that the model part of the cost function (eq. 6) is dimensionless. The constant of proportionality, that is, the square of the given percentage, is taken around 0.1 .

The data covariance matrix $\boldsymbol{C}_{\boldsymbol{D}}$ is taken as

$\left(C_{D}\right)_{i, j}=\sigma_{i}^{e} \sigma_{j}^{e} \exp \left\{-\frac{\left|t_{i}-t_{j}\right|}{\xi}\right\}$,

where $\sigma_{i}^{e}$ is the effective standard deviation of the $i^{\text {th }}$ data, defined as

$\sigma_{i}^{e}=\sqrt{\frac{\Delta t}{2 \xi}} \sigma\left(t_{i}\right)$.

$\sigma\left(t_{i}\right)$ is the standard deviation of the data at the time $t_{i}, \Delta t$ is the time step and $\xi$ is a smoothing period. Each data sample is exponentially correlated with its neighbours. Expression (8) amounts to introducing a $\mathbf{H}^{1}$ norm for the data into the cost function (eq. 6). Considering the whole signal with the covariance kernel of eq. (8), where the times $t_{i}$ and $t_{j}$ are between $t_{1}$ and $t_{n}$, we can show that the corresponding $\|\mathbf{d}\|_{D}^{2}$ cost function part in eq. (6) is given by (see, e. g. Tarantola 1987 , p. 572-576)

$$
\begin{aligned}
\|\mathbf{d}\|_{D}^{2}= & \sum_{i=1}^{n}\left(\frac{d_{i}}{\sigma\left(t_{i}\right)}\right)^{2}+\left(\frac{\xi}{\Delta t}\right)^{2} \sum_{i=1}^{n}\left(\frac{\Delta t}{\sigma\left(t_{i}\right)} \frac{\mathrm{d} d}{\mathrm{~d} t}\left(t_{i}\right)\right)^{2} \\
& +\left(\frac{\xi}{\Delta t}-\frac{1}{2}\right)\left(\left(\frac{d_{1}}{\sigma\left(t_{1}\right)}\right)^{2}+\left(\frac{d_{n}}{\sigma\left(t_{n}\right)}\right)^{2}\right)
\end{aligned}
$$

This shows that the cost function essentially corresponds to the common least-squares term complemented by an additional term controlling the derivative of the signal, which vanishes with $\xi / \Delta t$. This is equivalent to adjusting both the particle displacement and particle velocity, a trick that incorporates more information on the phase of the waveforms. Parameter $\xi$ determines whether the inversion is dominated by the fit of the particle displacement $(\xi<\Delta t)$ or by the fit of the particle velocity $(\xi>\Delta t)$ in the $L_{2}$-norm sense. In practice, and in the following examples, $\xi$ is kept around $\Delta t$, and $\sigma\left(t_{i}\right)$ is assigned a percentage of the signal $d_{i}$. This implies that the cost function $S(\mathbf{m})$ (eq. 6) is dimensionless and that the data and model terms can be directly compared.

Our choice to use a quasi-Newton algorithm to minimize the misfit function $S(\mathbf{m})$ is justified by the small size of the model space when considering layered media. This procedure was found to be more efficient than the conjugate gradient algorithm in terms of convergence rate and accuracy of results (De Barros \& Dietrich 2007). Thus, the model at iteration $n+1$ is obtained from the model at iteration $n$ by following the direction of pre-conditioned steepest descent defined by

$\mathbf{m}_{n+1}-\mathbf{m}_{n}=-\boldsymbol{H}_{n}^{-1} \cdot \boldsymbol{\gamma}_{n}$,

where $\boldsymbol{\gamma}_{n}$ is the gradient of the misfit function with respect to the model properties $\mathbf{m}_{n}$. Gradient $\boldsymbol{\gamma}_{n}$ can be expressed in terms of the Fréchet derivatives $\boldsymbol{F}_{n}$ and covariance matrices $\boldsymbol{C}_{\boldsymbol{M}}$ and $\boldsymbol{C}_{\boldsymbol{D}}$ (Tarantola 1987)

$\boldsymbol{\gamma}_{n}=\left.\frac{\partial S}{\partial \mathbf{m}}\right|_{\mathbf{m}_{n}}=\boldsymbol{F}_{n}^{T} \boldsymbol{C}_{\boldsymbol{D}}^{-1}\left(\mathbf{d}_{n}-\mathbf{d}_{\mathrm{obs}}\right)+\boldsymbol{C}_{\boldsymbol{M}}^{-1}\left(\mathbf{m}_{n}-\mathbf{m}_{\mathrm{prior}}\right)$.

In eq. (11), $\boldsymbol{H}_{n}$ is the Hessian of the misfit function, that is, the derivative of the gradient function, or the second derivative of the misfit function with respect to the model properties. We use the classical approximation of the Hessian matrix in which the second-order derivatives of $f(\mathbf{m})$ are neglected. This leads to a quasi-Newton algorithm equivalent to the iterative least-squares algorithm of Tarantola \& Valette (1982). The Hessian of the misfit function for $\mathbf{m}_{n}$ is then approximated by

$\boldsymbol{H}_{n}=\left.\frac{\partial^{2} S}{\partial \mathbf{m}^{2}}\right|_{\mathbf{m}_{n}} \simeq \boldsymbol{F}_{n}^{T} \boldsymbol{C}_{\boldsymbol{D}}{ }^{-1} \boldsymbol{F}_{n}+\boldsymbol{C}_{\boldsymbol{M}}{ }^{-1}$.

As the size of the Hessian matrix is relatively small with the 1-D model parametrization adopted for laterally homogeneous media, we keep the full expression given above and do not use further approximations of the Hessian by considering diagonal or blockdiagonal representations. Since the Hessian matrix is symmetric and positive definite, it can be inverted by means of a Cholesky decomposition. We find it more efficient to use a conjugate gradient algorithm with a pre-conditionning by the Cholesky decomposition to solve more accurately the system corresponding to eq. (11).

To illustrate the computation of the Fréchet derivatives $\boldsymbol{F}$ for a given model $\mathbf{m}$, we detail below, in the $P-S V$ case, the expressions of the first-order derivatives of displacement $U$ with respect to the solid density $\rho_{s}$ and permeability $k_{0}$. The following analytical formulae were derived in the frequency $\omega$ and ray parameter $p$ domain by considering a seismic source located at depth $z_{S}$, a model perturbation at depth $z$ and a receiver at depth $z_{R}$ (De Barros \& Dietrich 2008):

$$
\begin{aligned}
& \frac{\partial U\left(z_{R}, \omega ; z_{S}\right)}{\partial \rho_{S}(z)}=-\omega^{2}(1-\phi)\left[U G_{1 z}^{1 z}+V G_{1 z}^{1 r}\right], \\
& \frac{\partial U\left(z_{R}, \omega ; z_{S}\right)}{\partial k_{0}(z)}=\frac{\omega \eta}{k_{0}^{2} \Omega}\left(2 \frac{\omega}{\omega_{c}}+i n_{J}\right)\left[W G_{1 z}^{2 z}+X G_{1 z}^{2 r}\right] .
\end{aligned}
$$

In these expressions, $U=U\left(z, \omega ; z_{S}\right), V=V\left(z, \omega ; z_{S}\right), W=$ $W\left(z, \omega ; z_{S}\right)$ and $X=X\left(z, \omega ; z_{S}\right)$ denote the incident wavefields at the level of the model perturbation. $U$ and $V$, respectively, represent the vertical and radial components of the solid displacements; $W$ and $X$ stand for the vertical and radial components of the relative fluid-to-solid displacements. Expressions $G_{i j}^{k l}=G_{i j}^{k l}\left(z_{R}, \omega ; z\right)$ represent the Green's functions conveying the scattered wavefields from the inhomogeneities to the receivers. $G_{i j}^{k l}\left(z_{R}, \omega, z_{S}\right)$ is the Green's function corresponding to the displacement at depth $z_{R}$ of phase $i$ (values $i=1,2$ correspond to solid and relative fluid-to-solid motions, respectively) in direction $j$ (vertical $z$ or radial $r$ ) generated by a harmonic point force $F_{k l}\left(z_{S}, \omega\right)(k=1,2$ corresponding to a stress discontinuity in the solid and to a pressure gradient in the fluid, respectively) at depth $z_{S}$ in direction $l$ ( $z$ or $r$ ). A total of 16 different Green's functions are needed to express the four displacements $U, V, W$ and $X$ in the $P-S V$-wave system (4 displacements $\times 4$ forces). The $\Omega$ parameter appearing in derivative $\partial U / \partial k_{0}$ is defined by $\Omega=\sqrt{n_{J}-4 i \omega / \omega_{c}}$.

Fig. 2 shows the gradient $\boldsymbol{\gamma}_{0}$, the Hessian $\boldsymbol{H}_{0}$ and the term $-\boldsymbol{H}_{0}^{-1} \cdot \boldsymbol{\gamma}_{0}$ at the first iteration of the inversion of the solid density $\rho_{s}$ for a 20 -layer model. We note that the misfit function is mostly sensitive to the properties of the shallow layers of the model. However, we see that the term $-\boldsymbol{H}_{0}^{-1} \cdot \boldsymbol{\gamma}_{0}$ used in the quasi-Newton approach results in an enhanced sensitivity to the deepest layers, and therefore, in an efficient updating of their properties during inversion. As shown by Pratt et al. (1998), the inverse of the Hessian matrix plays an important role to scale the steepest-descent directions, since it partly corrects the geometrical spreading.

As the forward modelling operator $f$ is non-linear, several iterations are necessary to converge towards the global minimum 


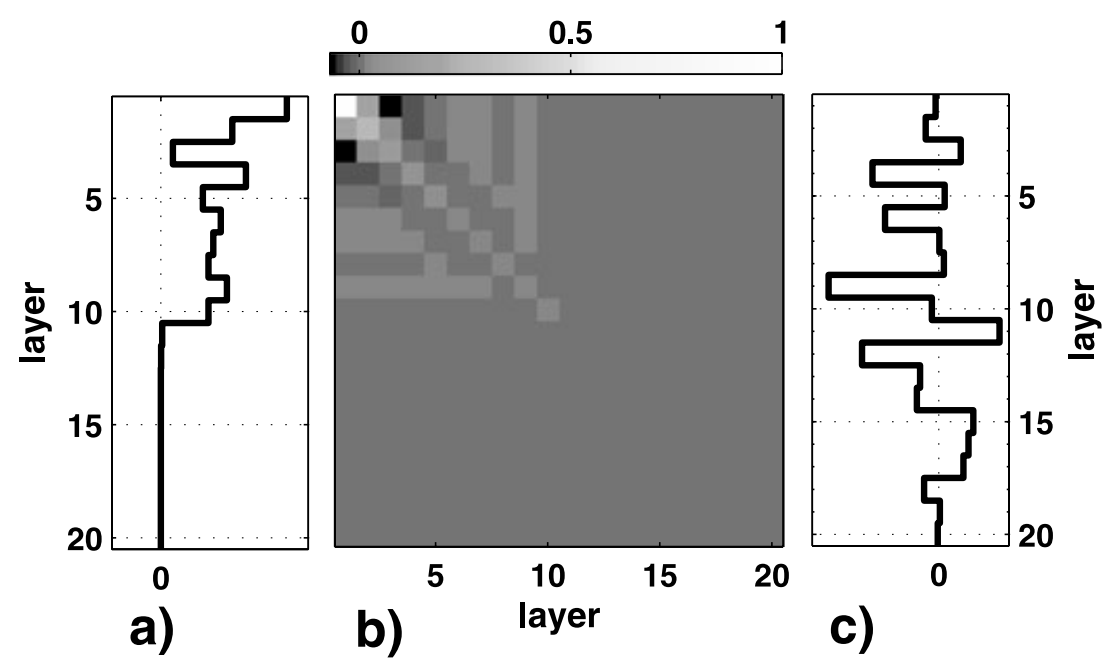

Figure 2. (a) Gradient $\boldsymbol{\gamma}_{0}$; (b) Hessian matrix $\boldsymbol{H}_{0}$ of the misfit function and (c) term $-\boldsymbol{H}_{0}^{-1} \cdot \boldsymbol{\gamma}_{0}$ at the first iteration of the inversion of the solid density for a 20-layer model.

solution provided that the a priori model is close enough to the true model. To ensure convergence of the iterative process, a coefficient $\epsilon_{n} \leq 1$ is introduced in eq. (11), such as

$\mathbf{m}_{n+1}-\mathbf{m}_{n}=-\epsilon_{n} \cdot \boldsymbol{H}_{n}^{-1} \cdot \boldsymbol{\gamma}_{n}$.

If the misfit function $S(\mathbf{m})$ fails to decrease between iterations $n$ and $n+1$, the value of $\epsilon_{n}$ is progressively reduced to modify model $\mathbf{m}_{n+1}$ until $S\left(\mathbf{m}_{n+1}\right)<S\left(\mathbf{m}_{n}\right)$. The inversion process is stopped when the misfit function becomes less than a pre-defined value or when a minimum of the misfit function is reached.

\section{ONE-PARAMETER INVERSION : CHECKING THE ALGORITHM}

\subsection{One-parameter inversion}

To determine the accuracy of the inversion procedure for the different model parameters considered, we first invert for a single parameter, in this case the mineral density $\rho_{s}$, and keep the others constant. The true model to reconstruct and the initial model used to initialize the iterative inversion procedure (which is also the a priori model) are displayed in Fig. 3. The other parameters are assumed to be perfectly known. Their vertical distributions consist of four 250-m thick homogeneous layers. Parameters $\phi, c_{s}$ and $k_{0}$ decrease with depth while parameters $\rho_{f}, K_{s}, K_{f}$ and $G_{s}$ are kept strictly constant.

Vertical-component seismic data (labelled DATA in the plots) are then computed from the true model for an array of 50 receivers spaced $20 \mathrm{~m}$ apart at offsets ranging from 10 to $1000 \mathrm{~m}$ from the source (Fig. 4). The latter is a vertical point force whose signature is a perfectly known Ricker wavelet with a central frequency of $25 \mathrm{~Hz}$. Source and receivers are located at the free surface. As mentioned previously, direct and surface waves are not included in our computations to avoid complications associated with these contributions. Fig. 4 also shows the seismograms (labelled INIT) at the beginning of the inversion, that is, the seismograms which are computed from the starting model. In this example, a minimum of the misfit function was reached after performing 117 iterations during which the misfit function was reduced by a factor of 2500 (Fig. 5). The decrease of the cost function is very fast during the first iterations and slows down subsequently. Fig. 3 shows that the

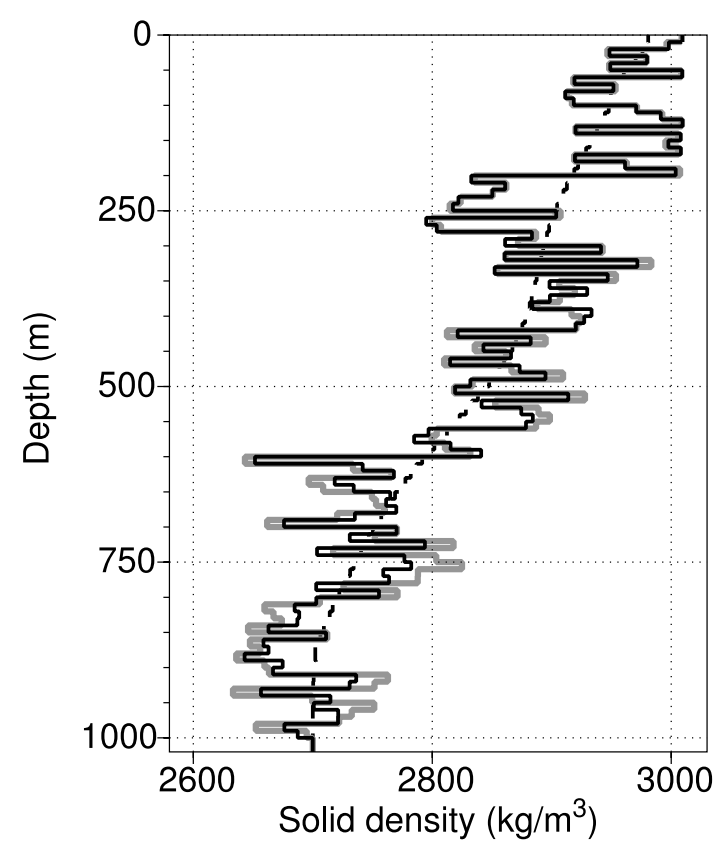

Figure 3. Models corresponding to the inversion for the mineral density $\rho_{s}$ : initial model, which is also the a priori model (dashed line), true model (thick grey line) and final model (black line). The corresponding seismograms are given in Fig. 4.

true model is very accurately reconstructed by inversion. As there are no major reflectors in the deeper part of the model, very little energy is reflected towards the surface, which leads to some minor reconstruction problems at depth. In Fig. 4, we note that the final synthetic seismograms (SYNT) almost perfectly fit the input data (DATA) as shown by the data residuals (RES) which are very small.

The inversions carried out for the $\phi, \rho_{f}, K_{s}, K_{f}, G_{s}$ and $c_{s}$ parameters (not shown) exhibit the same level of accuracy. However, as predicted by De Barros \& Dietrich (2008) and Morency et al. (2009) with two different approaches, the weak sensitivity of the reflected waves to the permeability does not allow us to reconstruct the variations of this parameter. 

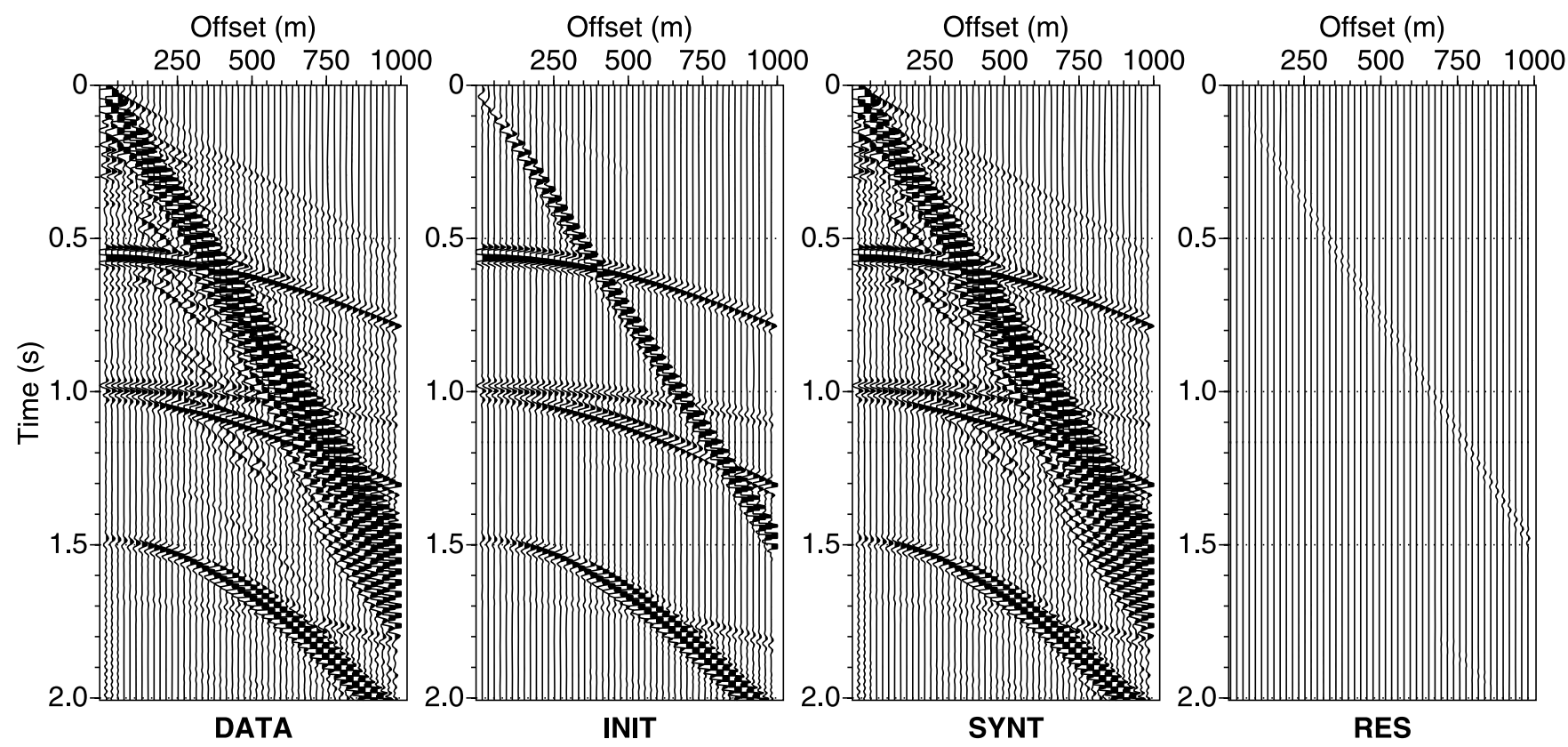

Figure 4. Seismograms corresponding to the inversion for the mineral density $\rho_{s}$ : synthetic data used as input (DATA), seismograms associated with the initial model (INIT), seismograms obtained at the last iteration (SYNT) and data residuals (RES) computed from the difference between the DATA and SYNT sections for the models depicted in Fig. 3. For convenience, all sections are displayed with the same scale, but the most energetic signals are clipped.

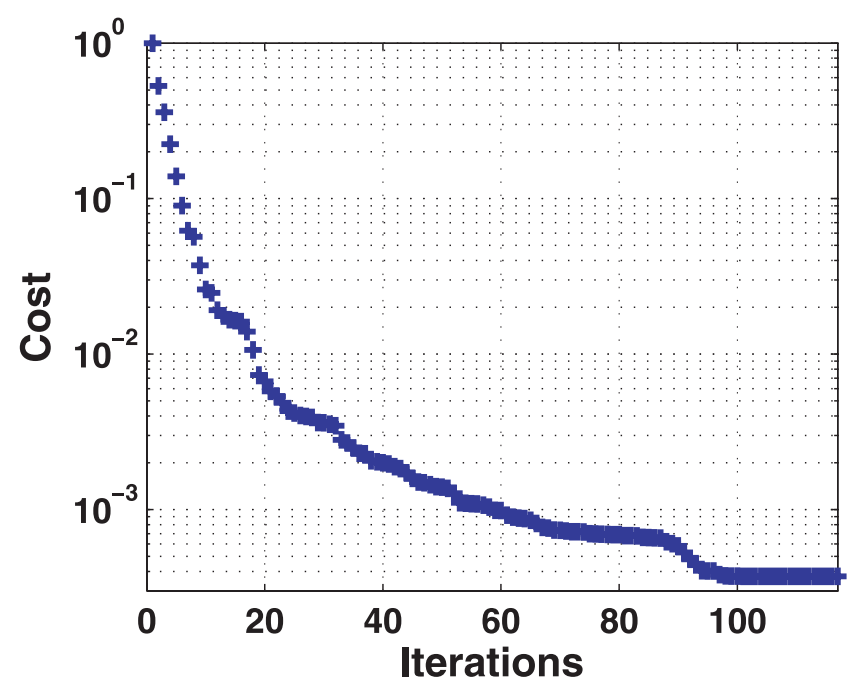

Figure 5. Decrease of the cost function (eq. 6) versus the number of iterations in the inversion of the solid density (Figs 3 and 4).

\subsection{Inversion resolution and accuracy}

To further study the application of the inversion method to field data, we examine in this section the resolution and accuracy issues of the inversion algorithm. The resolution of the reconstructed models depends on the frequency content of the seismic data. We have verified that our inversion procedure is capable of reconstructing the properties of layers whose thickness is greater than $\lambda / 2$ to $\lambda / 4$, where $\lambda$ is the dominant wavelength of the seismic data. This behaviour is similar to the results obtained by Kormendi \& Dietrich (1991) in the elastic case. Since $S$ waves generally have shorter wavelengths than $P$ waves, we would expect to obtain better model resolution by inverting only $S$ waves in a $S H$ data acquisition configuration, rather than using both $P$ and $S$ waves. However, the simultaneous inversion of $P$ and $S$ waves yields better inversion results due to the integration of information coming from both wave types.

In the inversion results presented in Figs 3 and 4, the thickness of the elementary layers used to discretize the subsurface is constant and equal to $10 \mathrm{~m}$, and the interfaces of the true models are correctly located at depth. In reality, the exact locations of the major interfaces are unknown and do not necessarily coincide with the layering defined in the subsurface representation, unless the model is very finely stratified. To evaluate the effect of a misplacement of the interfaces on the inversion results, we consider again the inversion of the solid density shown in Figs 3 and 4, and introduce a 1 per cent shift of the interface depths relative to the true model. This error may represent a displacement of $\sim \lambda / 20$ for the deepest interfaces. The inversion then leads to computed seismograms that correctly fit the input data, and to a final model very close to the true model. Therefore, this test shows that the inversion algorithm can tolerate small errors affecting the interface depths. It also indicates that a discretization of the order of $\lambda / 20$ should be used to represent the layering of the subsurface.

The accurate reconstruction of the distributions of model parameters requires large source-receiver offset data to reduce various ambiguities inherent to the inversion procedure. However, large-offset data do not always constrain the solution as expected because of the strong interactions of the wavefields with the structures at oblique angles of incidence. In particular, large-offset data may contain energetic multiple reflections which increase the non-linearity of the inverse problem. Therefore, a trade-off must be found in terms of source-receiver aperture to improve the estimation of model parameters while keeping the non-linearity of the seismic response at a reasonable level. Our tests indicate that the layered models are badly reconstructed if the structures are illuminated with angles of incidence smaller than $10^{\circ}$ relative to the normal to the interfaces. Conversely, the inversion procedure generally yields good results for incidence angles greater than $45^{\circ}$, a value not always reached in conventional seismic reflection surveys. As the medium to reconstruct is laterally homogeneous (i.e. invariant by translation), 
the inversion process requires only one source and does not require a very fine spatial sampling of the wavefields along the recording surface to work properly. For the example given in Figs 3 and 4, 25 traces were sufficient to correctly reconstruct the model properties down to $1000 \mathrm{~m}$ provided that the maximal source-receiver offset be greater than $1000 \mathrm{~m}$.

A classical approach to address the inversion of backscattered waves is to gradually incorporate new data during the inversion process rather than using the available data all together. A common strategy followed by many authors (for instance Kormendi \& Dietrich 1991; Gélis et al. 2007) for seismic reflection data is to partition the data sets according to source-receiver offset and recording time, and possibly temporal frequency. The inversion is then carried out in successive runs, by first including the early arrivals, near offsets and low frequencies, and by integrating additional data corresponding to late arrivals, large offsets and higher frequencies in subsequent stages. This strategy is implemented to obtain stable inversion results by first determining and consolidating the gross features (long and intermediate wavelengths) of the upper layers before estimating the properties of the deepest layers and the fine-scale details (short wavelengths) of the structure. We have checked for a complex medium and single parameter inversions that this progressive and cautious strategy leads to better results than the straightforward and indistinct use of the whole data set.

\section{MULTIPARAMETER INVERSION: PARAMETER COUPLING}

\subsection{Model parameters for the inversion of synthetic data}

In their sensitivity study of poroelastic media, De Barros \& Dietrich (2008) showed that perturbations of certain model parameters (especially $c_{s}, \phi$ and $G_{s}$ on the one hand, and $\rho_{s}$ and $\rho_{f}$ on the other hand) lead to similar seismic responses, thus emphasizing the strong coupling of these model parameters. In such cases, a multiparameter inversion is not only challenging, but may be impossible to carry out, except for very simple model parameter distributions, such as ‘boxcar functions' (De Barros \& Dietrich 2007).

We consider simple models to specifically address this issue. The data sets used in this section are constructed from a generic twolayer model (see Table 2) inspired by an example given in Haartsen \& Pride (1997): a 100-m thick layer (with properties typical of consolidated sand) overlying a half-space (with properties typical of sandstone). The permeability, porosity and consolidation parameter differ in the two macrolayers whereas the five other model parameters have identical values. This generic model is then subdivided into 20 elementary layers having a constant thickness of $10 \mathrm{~m}$. One or several properties chosen among the eight model parameters are then randomly modified in the elementary layers. The models thus obtained are used to compute synthetic data constituting the input seismic waveforms for our inversion procedure.

The data were computed for 50 receivers distributed along the free surface, between 10 and $500 \mathrm{~m}$ from the source location. The seismic source is a vertical point force, with a $45-\mathrm{Hz}$ Ricker wavelet source time function.

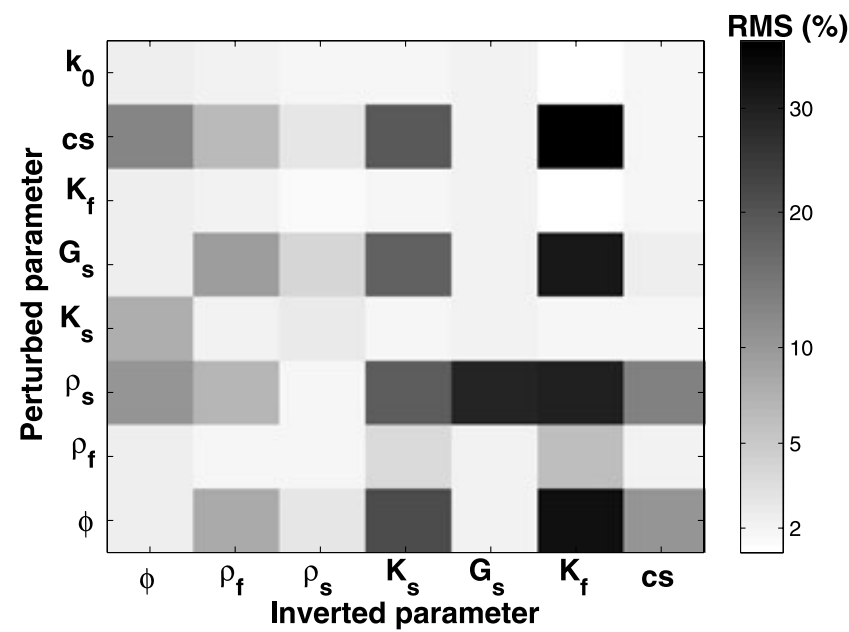

Figure 6. Normalized rms error of model parameter logs obtained by inversion using an incorrect starting model. The parameters which are inverted are indicated along the horizontal axis, whereas the parameters which are perturbed in the starting model are along the vertical axis. The starting model is not perturbed when the parameters along the horizontal and vertical axes are identical. Permeability $k_{0}$ was not inverted in this test.

\subsection{Coupling between parameters}

We seek to know if a given parameter can be correctly reconstructed if one of the other model properties is imperfectly known. For each parameter considered, we evaluate the robustness of the inversion when a single parameter of the a priori and initial model (given in Table 2 ) is modified by +1 per cent everywhere in the model. This variation does not pretend to be realistic, but is merely introduced to evaluate the coupling between parameters. To obtain a direct assessment of the resulting discrepancies, we measure the normalized rms error between the reconstructed parameters $\boldsymbol{m}$ and their true values $\boldsymbol{m}_{\boldsymbol{t}}$ :

$E_{\mathrm{rms}}=\sqrt{\frac{\sum_{n}\left[\boldsymbol{m}(n)-\boldsymbol{m}_{t}(n)\right]^{2}}{\sum_{n}\left[\boldsymbol{m}_{t}(n)\right]^{2}}}$,

where $n$ represents any element of the model parameter arrays $\boldsymbol{m}$ and $\boldsymbol{m}_{\boldsymbol{t}}$.

The results of this test are summarized in Fig. 6 where the inverted parameters are along the horizontal axis and the perturbed parameters are along the vertical axis. Fig. 6 displays uneven results where the reconstruction of some parameters (notably $\phi, \rho_{f}, \rho_{s}$ and $c_{s}$ ) is only moderately affected by the small perturbations applied to other model properties, while the inversion of other parameters $\left(K_{f}\right.$, $K_{s}$ and $G_{s}$ ) is strongly influenced by errors in single model properties. The rms errors greater than 10 per cent usually correspond to reconstructed models which are very far from reality. Note that no perturbation has been introduced in the model when parameters are identical along both axes. In those cases, the inversion is not perfect (model errors may reach 2 per cent) as there exists minor differences in the deepest layers due to a lack of seismic information contained in the seismograms. This sensitivity study shows that parameters are poorly determined if the initial and a priori models of the other

Table 2. Model parameters of the generic two-layer model used in the inversion tests.

\begin{tabular}{|c|c|c|c|c|c|c|c|c|c|}
\hline Depth (m) & $\phi()$ & $k_{0}\left(\mathrm{~m}^{2}\right)$ & $\rho_{f}\left(\mathrm{~kg} \mathrm{~m}^{-3}\right)$ & $\rho_{s}\left(\mathrm{~kg} \mathrm{~m}^{-3}\right)$ & $K_{s}(\mathrm{GPa})$ & $G_{S}(\mathrm{GPa})$ & $K_{f}(\mathrm{GPa})$ & $c_{S}()$ & $\eta(\mathrm{Pa} \mathrm{s})$ \\
\hline $0-100$ & 0.30 & $10^{-11}$ & 1000 & 2700 & 36 & 40 & 2.2 & 16.5 & 0.001 \\
\hline $100-\infty$ & 0.15 & $10^{-13}$ & 1000 & 2700 & 36 & 40 & 2.2 & 9.5 & 0.001 \\
\hline
\end{tabular}


parameters are not perfectly known, and as a result, multiparameter inversion results must in general be considered with utmost care.

As predicted by the sensitivity study conducted by De Barros \& Dietrich (2008), the seismic response of a poroelastic medium, and therefore the inversion of this response are mostly sensitive to parameters $\rho_{s}, \phi, c_{s}$ and $G_{s}$. In Fig. 6 , the horizontal lines corresponding to these parameters display the largest errors. It is seen that the inversions of the bulk moduli $K_{s}$ and $K_{f}$ are unstable if the four parameters mentioned above are not well defined in the starting model. We also note that the inversion for $G_{s}$ is only sensitive to $\rho_{s}$, and that the inversion for $\phi$ or $c_{s}$ does not depend on $G_{s}$. On the other hand, a poor knowledge of the permeability $k_{0}$, of the fluid modulus $K_{f}$ or of the fluid density $\rho_{f}$ in the starting model has little or no influence on the inversion of the other parameters. Among the four most sensitive parameters, $\rho_{s}$ and $G_{s}$ can usually be estimated with good accuracy from geological knowledge. In addition, these parameters do not show large variations. Consequently, $\phi$ and $c_{s}$ are the most important parameters to estimate in the starting model for a successful inversion of the medium properties.

\subsection{Multiparameter inversion}

By definition and construction of the full waveform fitting procedure, the observed data are generally well reproduced by the synthetics at the end of the inversion process. However, when parameters are coupled, the resulting models are wrong even though the corresponding waveforms appear to properly represent the data. For example, Fig. 7 shows the models obtained by the simultaneous inversion of two strongly coupled parameters, namely, porosity $\phi$ and consolidation parameter $c_{s}$. The corresponding data are shown in Fig. 8. It is seen that in a given layer, the error made on one parameter is partly compensated by an error of opposite sign on the other parameter, thereby minimizing the misfit function. The impossibility to simultaneously reconstruct several model parameters at the same time means that the extra information introduced by the peculiarities of the seismic wave propagation in poroelastic media (slow $P$ wave, conversion of the fluid displacement into solid displacement and attenuation and dispersion effects) is not sufficient, for the source-receiver geometry under consideration, to overcome the intrinsic ambiguities of the poroelastic model and its description in terms of eight material properties.

\section{INVERSION STRATEGIES}

We explore in this section two strategies to get around the parameter coupling, by first considering composite model parameters and $a$ priori information, and then a differential inversion procedure suited for time-lapse surveys.

\subsection{Composite model parameters}

The first strategy to circumvent the difficulty of multiparameter inversion is to use as much external information as possible and to combine model parameters that are physically interdependent. In our approach, composite model parameters can be easily introduced in the inversion algorithm by combining the Fréchet derivatives of the original model parameters (see, e.g. De Barros \& Dietrich 2008).

For example, we may know from a priori information the nature of the two fluids saturating a porous medium, for example, water and air, and assume standard values for the properties of each fluid, for example, $K_{f}=2.1 \mathrm{GPa}$ and $\rho_{f}=1000 \mathrm{~kg} \mathrm{~m}^{-3}$ for water. We can then introduce the fluid saturation rate $S_{r}$ as the ratio of the volume $V_{f 1}$ occupied by the more viscous fluid to the total pore volume $V_{f}$. The sensitivity operators with respect to the fluid saturation rate are computed by using mixture laws, that is, by using an arithmetic average (Voigt's law) for the density and a harmonic average (Reuss's law) for the fluid modulus,

$S_{r}=\frac{V_{f 1}}{V_{f}}=\frac{\rho_{f}-\rho_{f 2}}{\rho_{f 1}-\rho_{f 2}}=\frac{K_{f 1}}{K_{f}} \frac{K_{f}-K_{f 2}}{K_{f 1}-K_{f 2}}$,

where subscripts 1 and 2 denote the properties of the two fluids. We can similarly define the volume rate $T_{s}$ of a mineral for a medium consisting of two minerals, as the volume occupied by one of the minerals normalized by the total solid volume. As before, we use Voigt's law to link up this parameter with the solid density and Reuss's law for the shear and bulk moduli. These mixture laws are not the most accurate ones to describe the properties of biphasic fluids or bicomponent minerals (Mavko et al. 1998), however, we will use them for sake of simplicity.

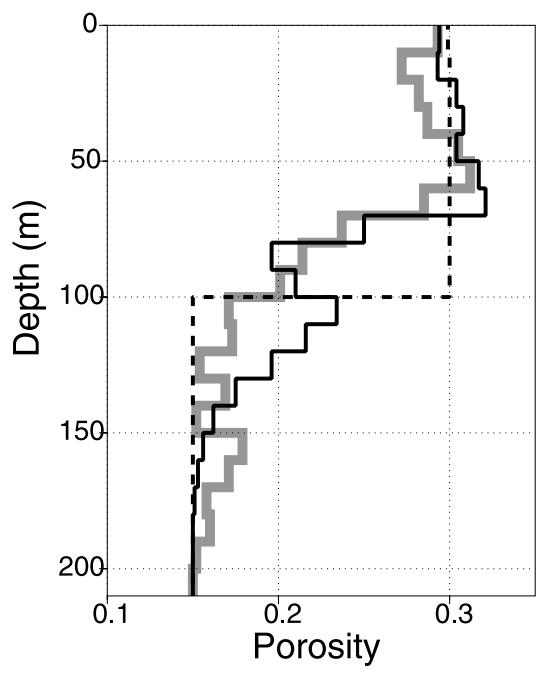

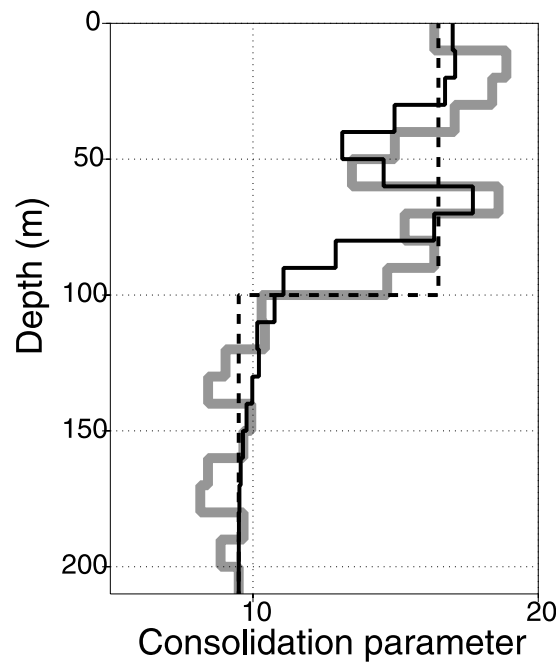

Figure 7. Models for the simultaneous inversion of the porosity (left-hand panel) and consolidation parameter (right-hand panel). Both panels show the initial model, which is also the a priori model (dashed line), the true model (thick grey line) and the final model (black line). The corresponding seismograms are displayed in Fig. 8. 

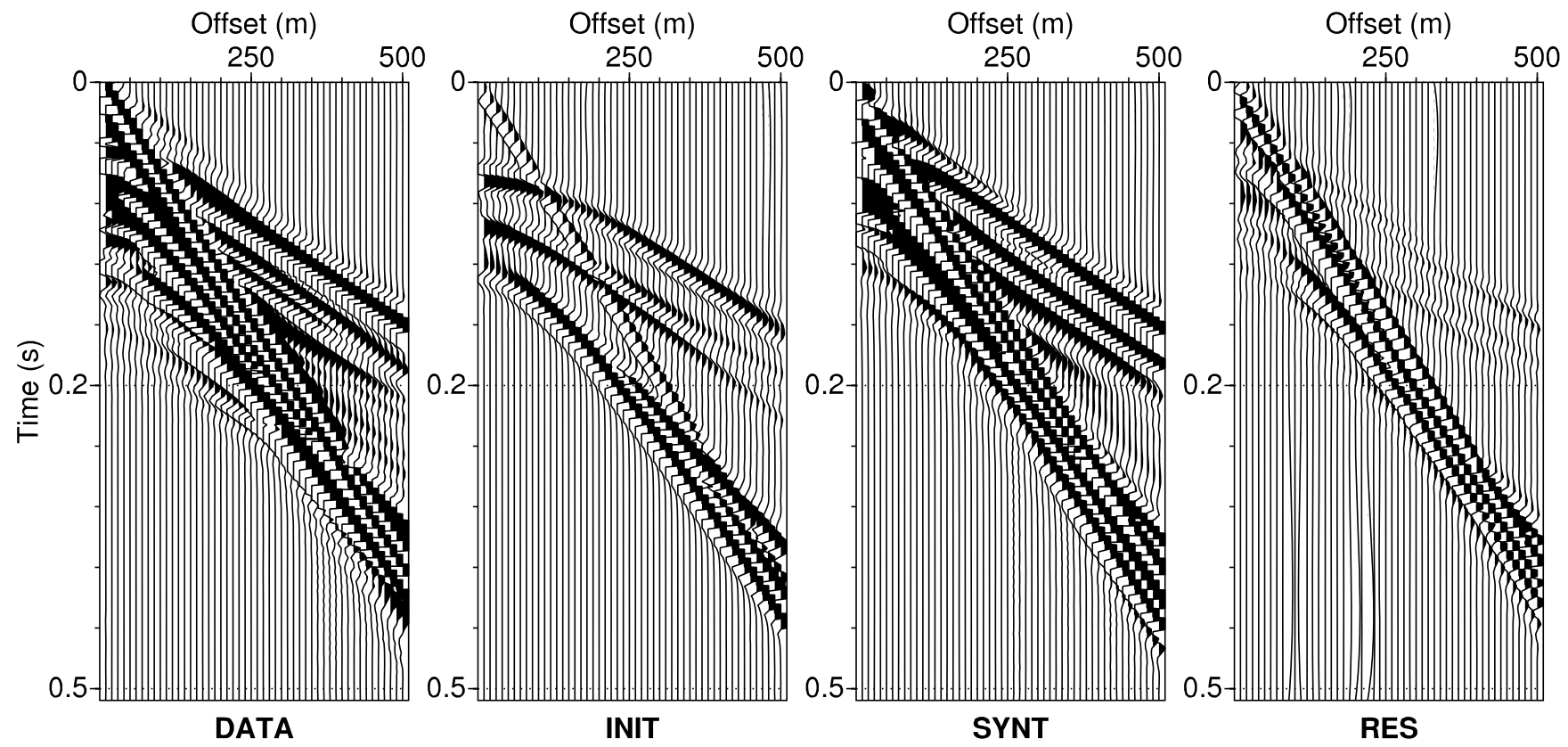

Figure 8. Seismograms corresponding to the models depicted in Fig. 7: synthetic data used as input (DATA), seismograms associated with the initial model (INIT), seismograms obtained at the last iteration (SYNT) and data residuals (RES) computed from the difference between the DATA and SYNT sections. For convenience, all sections are displayed with the same scale, but the most energetic signals are clipped.

We can then invert for the new composite parameter $T_{s}$ (resp. $S_{r}$ ) instead of considering density $\rho_{s}$ (resp. $\rho_{f}$ ) and moduli $K_{s}$ and $G_{s}$ (resp. $K_{f}$ ), by assuming that the other parameters are independently defined. Since the quantities $S_{r}$ and $T_{s}$ vary between 0 and 1 and are equipartitioned, care must be taken to ensure that these bounds are not exceeded. To satisfy these constraints, we introduce an additional change of variable to deal with Gaussian distribution parameters, as needed in the least-squares approach. Following Mora et al. (2006), and generically denoting the $S_{r}$ and $T_{s}$ parameters by variable $X$, we define a new parameter $X^{\prime}$ using the error function erf by writing

$$
X^{\prime}=\operatorname{erf}^{-1} \frac{2 X-X_{\max }-X_{\min }}{X_{\max }-X_{\min }}
$$

and

$\frac{\mathrm{d} X}{\mathrm{~d} X^{\prime}}=\frac{X_{\max }-X_{\min }}{\sqrt{\pi}} \mathrm{e}^{-X^{\prime 2}}$

In this way, the new parameter $X^{\prime}$ has a Gaussian distribution centred around 0 with a variance of $1 / 2$, which is equivalent in probability to an equipartition of the variable $X$ over the interval ]0,1[. The $X$ parameter $\left(T_{s}\right.$ or $\left.S_{r}\right)$ can never reach the values 0 or 1 as these values are infinite limits for the $X^{\prime}$ parameter. However, this limit can be accurately approached without any stability problems.

Fig. 9 shows an inversion example to obtain the variations of the volume rate of silica when the grains are constituted by silica $\left(K_{s}=\right.$ $36 \mathrm{GPa}, G_{s}=40 \mathrm{GPa}$ and $\left.\rho_{s}=2650 \mathrm{~kg} \mathrm{~m}^{-3}\right)$ and mica $\left(K_{s}=\right.$ $59.7 \mathrm{GPa}, G_{s}=42.3 \mathrm{GPa}$ and $\rho_{s}=3050 \mathrm{~kg} \mathrm{~m}^{-3}$ ). The other model parameters are those given in Table 2 which are assumed fixed and known. The unknowns of the inverse problem are the distributions of the $\rho_{s}, K_{s}$ and $G_{s}$ parameters characterizing the minerals, which are lumped together as the $T_{s}$ parameter. The source and receiver geometry and characteristics are the same as those described in Section 5.1. Fig. 10 displays the synthetic data computed from the models of Fig. 9, the seismograms at the last iteration of the

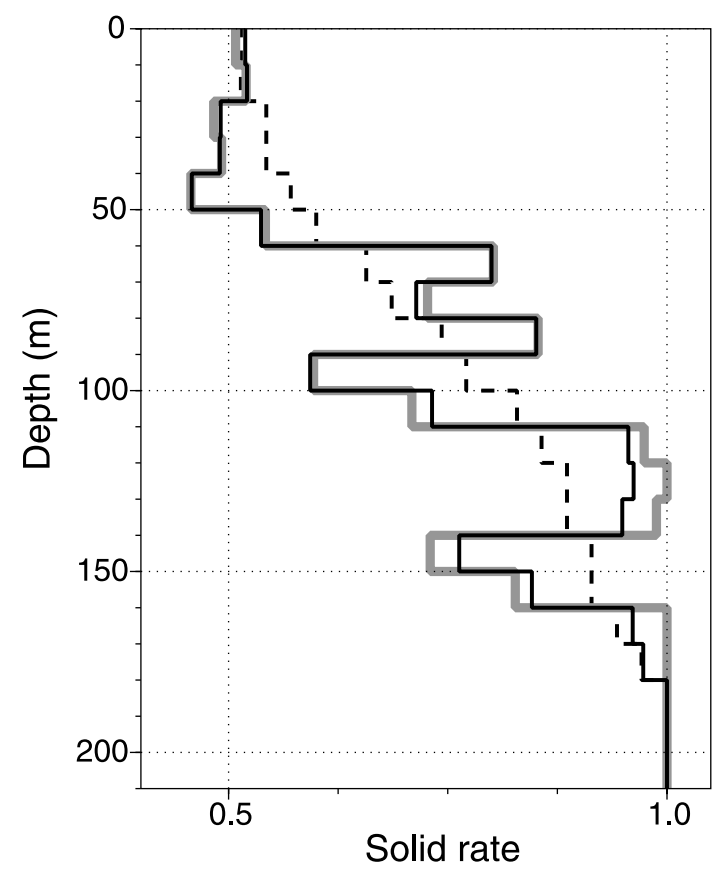

Figure 9. Models corresponding to the inversion for the volume rate of silica $T_{s}$ : initial model (dashed line), true model (thick grey line) and final model (black line). The corresponding seismograms are displayed in Fig. 10.

inversion process and the data residuals. Fig. 9 shows that the volume rate of silica is very well reconstructed. A total of 18 iterations were performed before reaching the minimum of the misfit function, which was divided by 950 during the inversion process.

These examples show that a viable inversion strategy is obtained by introducing (1) a priori informations (e.g. the type of fluids) and (2) a relationship between the former and the new parameters (e.g. relationships between $K_{f}, \rho_{f}$ and $S_{r}$ ). This approach 

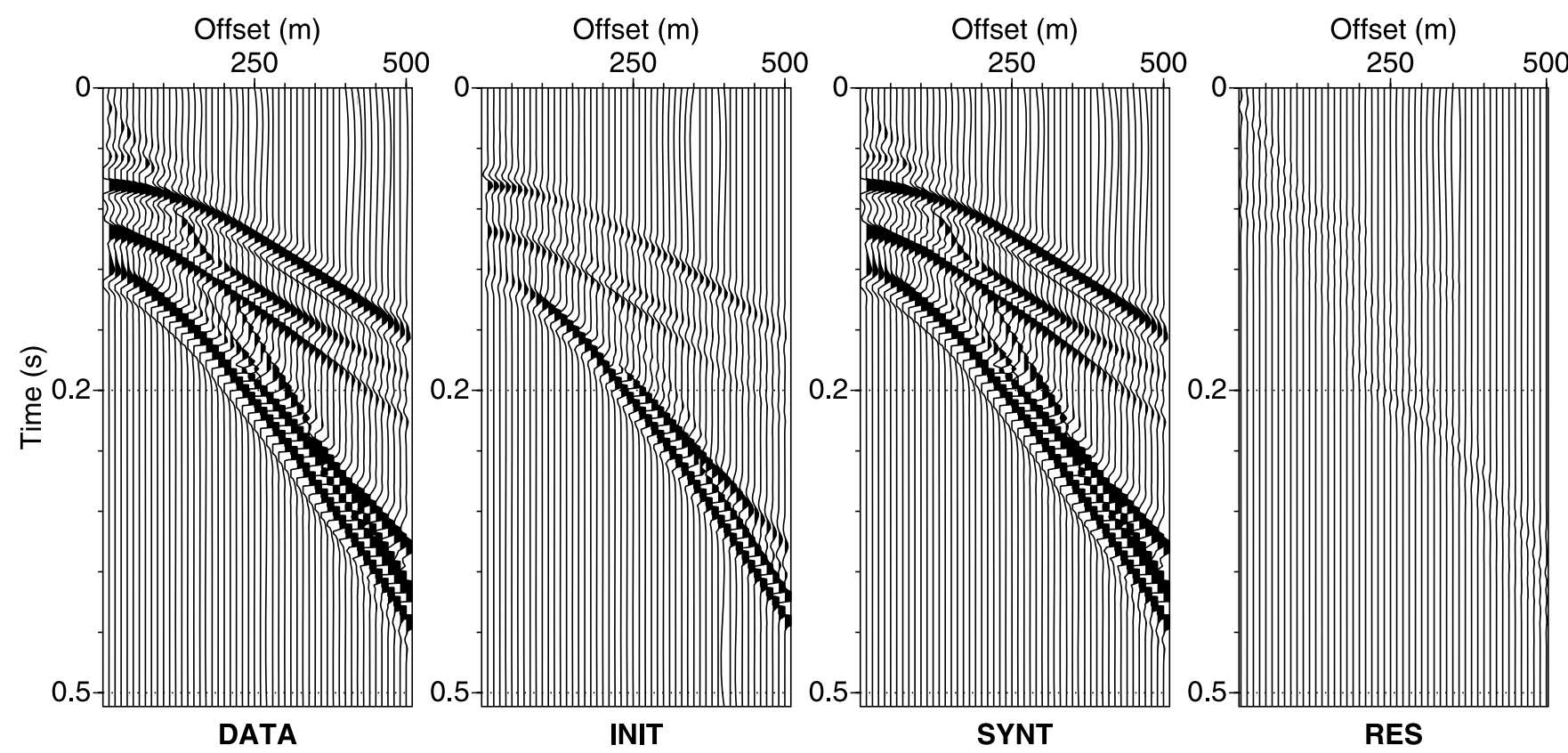

Figure 10. Seismograms corresponding to the models depicted in Fig. 9: synthetic data used as input (DATA), seismograms associated with the initial model (INIT), seismograms obtained at the last iteration (SYNT) and data residuals (RES) computed from the difference between the DATA and SYNT sections. For convenience, all sections are displayed with the same scale, but the most energetic signals are clipped.

can be generalized as soon as these two requirements are fulfilled. Following the same process, let us mention here that the number of parameters describing the medium can be further reduced if the properties characterizing the solid part of the porous medium (mineral properties $\rho_{s}, K_{s}$ and $G_{s}$, consolidation parameter $c_{s}$ and porosity $\phi$ ) can be measured via laboratory experiments. This may seem obvious but if the medium can be represented by two different facies (sand and shale, for example), we can invert for the volume percentage of each facies. In this case, the number of unknowns would be reduced to two: the type of facies (sand or shale), and its fluid content.

\subsection{Differential inversion}

A second possibility to reduce the ambiguities of multiparameter inversion is to consider and implement a differential inversion. Instead of dealing with the full complexity of the medium, we concentrate on small changes in the subsurface properties such as those occurring over time in underground fluid-filled reservoirs. This approach may be particularly useful for time-lapse studies to follow the extension of fluid plumes or to assess the fluid saturation as a function of time.

For example, the monitoring of underground $\mathrm{CO}_{2}$ storage sites mainly aims at mapping the expansion of the $\mathrm{CO}_{2}$ cloud in the subsurface and assessing its volume. Time-lapse studies performed over the Sleipner $\mathrm{CO}_{2}$ injection site in the North Sea (see e.g. Arts et al. 2004; Clochard et al. 2010) highlight the variations of fluid content as seen in the seismic data after imaging and inversion. In this fluid substitution case, the parameter of interest is the relative saturation of saline water/ $\mathrm{CO}_{2}$ although the fluid density is affected as well by the $\mathrm{CO}_{2}$ injection. We can then rearrange the model parameters to invert for the relative $\mathrm{H}_{2} \mathrm{O} / \mathrm{CO}_{2}$ saturation. A differential inversion process will allow us to free ourselves from the unknown model parameters, to a high degree. This approach is valid for any type of fluid substitution problem, such as water-table variation, oil and gas extraction or hydrothermal activity.
The first step in this approach is to perform a baseline or reference survey to estimate the solid properties before the fluid substitution occurs. We have shown in the section on multiparameter inversion that the model properties are poorly reconstructed in general (Fig. 7), whereas the seismic data are reasonably well recovered (Fig. 8). Thus, in spite of its defects, the reconstructed model respects to some degree the wave kinematics of the input data. In other words, the inverted model provides a description of the solid Earth properties which can be used as a starting model for subsequent inversions. The latter would be used to estimate the fluid variations within the subsurface from a series of monitor surveys (second step). To test this concept, we perturb the fluid properties of the true model of Fig. 7 to simulate a fluid variation over time. Two $30-\mathrm{m}$ thick layers located between 50 and $80 \mathrm{~m}$ depth and between 110 and $140 \mathrm{~m}$ depth are water depleted due to gas injection. The water saturation varies between 60 and 80 per cent in these two layers (Fig. 11). We assume that air is injected in the water, with properties $K_{f}=0.1 \mathrm{MPa}$ and $\rho_{f}=1.125 \mathrm{~kg} \mathrm{~m}^{-3}$ ). We consider air properties, even though is not corresponding to injection problems, to keep the problem as general as possible with regard to any kind of fluid substitution problem (involving $\mathrm{CO}_{2}$, gas, etc.). The starting models for the porosity $\phi$ and consolidation parameter $c_{s}$ are the ones reconstructed in Fig. 7. The initial seismograms (INIT) of Fig. 12 are the same as the output data (SYNT) of Fig. 8 (with different normalization). Our goal is to estimate the fluid properties by inverting the seismic data for the water saturation.

The model obtained is displayed in Fig. 11. We see that the location and extension of the gas-filled layers are correctly estimated. The magnitude of the water saturation curve, which defines the amount of gas as a function of depth, is somewhat underestimated in the top gas layer but is nevertheless reasonably well estimated. In the bottom gas layer, the inversion procedure only provides a qualitative estimate of the water saturation. The data residuals are quite strong in this example because they correspond to the sum of the residuals of both inversion steps. These computations show 


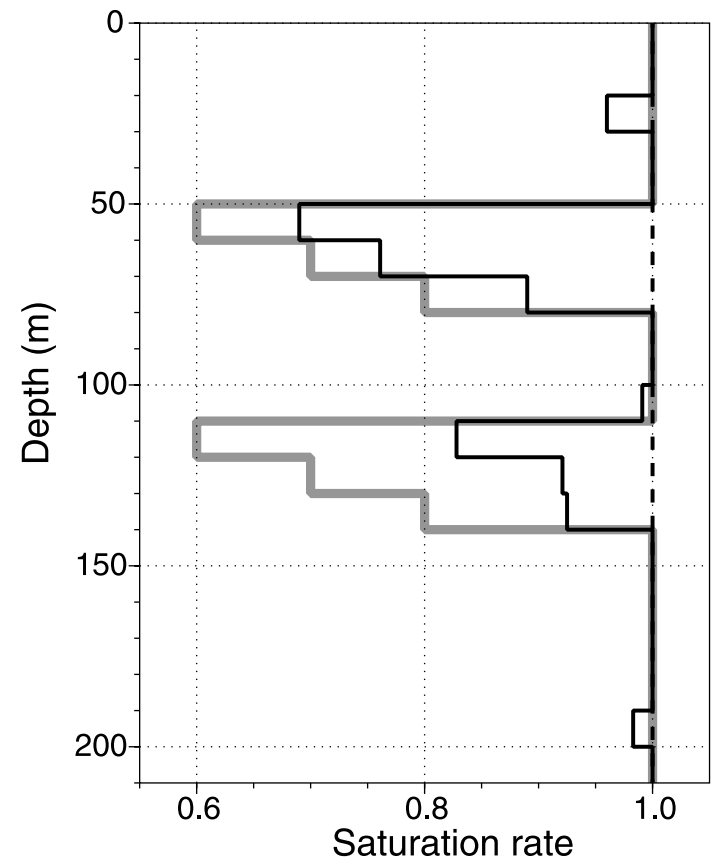

Figure 11. Models corresponding to the inversion for the water saturation $S_{r}$ : initial model (dashed line), true model (thick grey line) and final model (black line). The corresponding seismograms are given in Fig. 12.

that the differential inversion approach is capable of estimating the variations of fluid content in the subsurface without actually knowing the full properties of the medium.

\section{CONCLUSION}

We have developed a FWI technique with the aim of directly estimating the porosity, permeability, interstitial fluid properties and me- chanical parameters from seismic waveforms propagating in fluidfilled porous media. The inversion algorithm uses a conventional generalized least-squares approach to iteratively determine an earth model which best fits the observed seismic data. To investigate the feasibility of this concept, we have restricted our approach to planelayered structures. The forward problem is solved with the generalized reflection and transmission matrix method accounting for the wave propagation in fluid-filled porous media with the Biot theory. This approach is successfully checked against a semi-analytical solution. The input data of our inversion procedure consists of shot gathers, that is, backscattered energy recorded in the time-distance domain.

The numerical experiments carried out indicate that our inversion technique can - in favourable conditions-reproduce the fine details of complex earth models at reasonable computational times, thanks to the relatively fast convergence properties of the quasiNewton algorithm implemented. The inversion of a single model parameter yields very satisfactory results if large-aperture data are used, provided that the other parameters are well defined. As expected, the quality of the inversions mainly reflects the sensitivity of the backscattered wavefields to the different model parameters. The best results are obtained for the most influential parameters, namely, the porosity $\phi$, consolidation parameter $c_{s}$, solid density $\rho_{s}$ and shear modulus of the grains $G_{s}$. As a general rule, perturbations in fluid density $\rho_{f}$, solid bulk modulus $K_{s}$ and fluid bulk modulus $K_{f}$ have only a weak influence on the wave amplitudes. Permeability $k_{0}$ is the most poorly estimated parameter.

The number of model parameters entering the Biot theory (eight in our case) is not a problem by itself. However, the interdependence between some of these parameters is very challenging for the inversion since the information pertaining to one parameter may be wrongly transferred to another parameter. This is notably true for parameters $c_{s}, \phi$ and $G_{s}$ on the one hand, and for parameters $\rho_{s}$ and $\rho_{f}$ on the other hand. As a result, sequential or simultaneous inversions for several model parameters are usually impossible, in
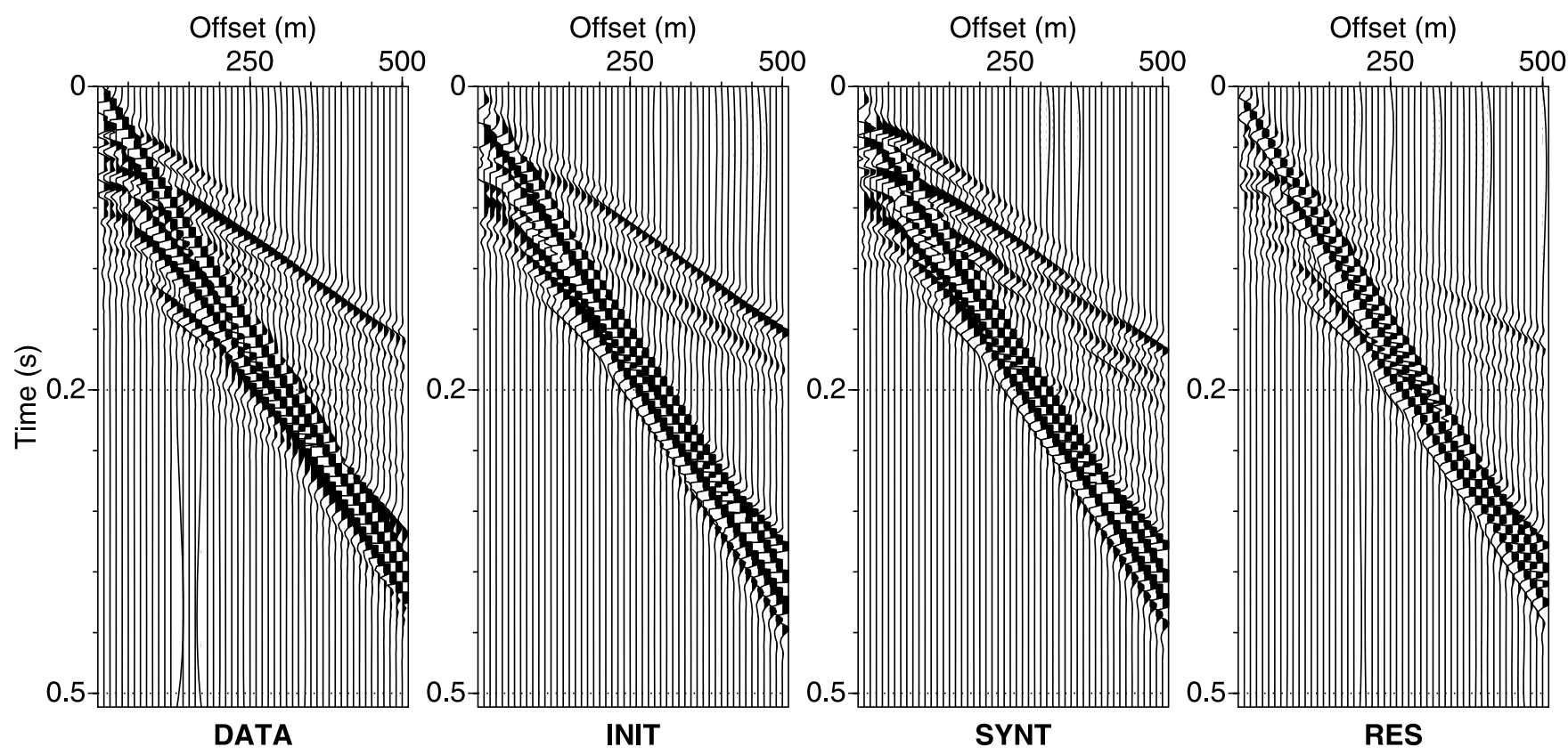

Figure 12. Seismograms corresponding to the models depicted in Fig. 11: synthetic data used as input (DATA), seismograms associated with the initial model (INIT), seismograms obtained at the last iteration (SYNT) and data residuals (RES) computed from the difference between the DATA and SYNT sections. For convenience, all sections are displayed with the same scale, but the most energetic signals are clipped. 
the sense that the models obtained are not reliable. The additional information carried by the waveforms propagating in poroelastic media does not help to better constrain the multiparameter inversion for the source-receiver configuration considered in this article.

Several strategies can be used to circumvent the coupling between the model parameters, either by using auxiliary information or by considering only perturbations in the medium properties over time. In the former case, the solution consists in defining composite parameters from the original medium properties, by using basic knowledge on the material properties. For example, a priori information on the nature of the saturating fluids can be exploited to invert for the fluid saturation rate. In other cases, we may consider inverting for the volume rate between two different lithologies if the rock formations are known beforehand from well log data. Our inversion algorithm can easily be modified to introduce new model parameters given that the Fréchet derivatives of the seismograms are expressed in semi-analytical form.

In time-lapse studies, such as for the seismic monitoring of underground $\mathrm{CO}_{2}$ storage, further simplifications occur as the fluid substitution problem only involves a limited number of material properties. The two-step approach proposed in this work for differential inversions of the reference and monitor surveys begins by estimating an optimum yet inaccurate earth model from the reference data set, which serves as a starting model for the inversion of the monitor data sets. This approach proved successful and promising for the reconstruction of the fluid saturation rate as a function of depth.

It is likely that 2-D or 3-D poroelastic inversions will lead to similar conclusions in terms of coupling between model parameters. Therefore, the most interesting use of such FWI algorithms will probably be in combination with conventional elastic inversions to estimate the wave velocities and bulk density first, and finish with the fine scale details of the poroelastic properties with a priori knowledge of some of the parameters, as suggested in this study.

\section{ACKNOWLEDGMENTS}

L. De Barros was partly funded by the department of Communications, Energy and Natural Resources (Ireland) under the National Geosciences programme 2007-2013. The relevant comments made by Christina Morency, the other reviewers and the editors of this paper greatly contributed to improve the manuscript. We are grateful to Florence Delprat-Jannaud, Stéphane Garambois, Patrick Lailly, Helle A. Pedersen and Gareth O'Brien for many helpful discussions in the course of this work. The numerical computations were performed by using the computer facilities of the Grenoble Observatory (OSUG).

\section{REFERENCES}

Arts, R., Eiken, O., Chadwick, A., Zweigel, P., des Meer, L.V. \& Zinszner, B., 2004. Monitoring of $\mathrm{CO}_{2}$ injected at Sleipner using time-lapse seismic data., Energy, 29, 1383-1392.

Auriault, J.-L., Borne, L. \& Chambon, R., 1985. Dynamics of porous saturated media, checking of the generalized law of Darcy, J. acoust. Soc. Am., 77(5), 1641-1650

Bachrach, R., 2006. Joint estimation of porosity and saturation using stochastic rock-physics modeling, Geophysics, 71(5), 53-63.

Ben-Hadj-Ali, H., Operto, S. \& Virieux, J., 2008. Velocity model-building by 3-D frequency-domain, full waveform inversion of wide aperture seismic data, Geophysics, 73(5), 101-117.

Berryman, J., Berge, P. \& Bonner, B., 2002. Estimating rock porosity and fluid saturation using only seismic velocities, Geophysics, 67, 391-404.
Biot, M., 1956. Theory of propagation of elastic waves in a fluid-saturated porous solid. I. Low-frequency range, II. Higher frequency range, $J$. acoust. Soc. Am., 28, 168-191.

Bosch, M., 2004. The optimization approach to lithological tomography: combining seismic data and petrophysics for porosity prediction, Geophysics, 69, 1272-1282.

Bouchon, M., 1981. A simple method to calculate Green's functions for elastic layered media, Bull. seism. Soc. Am., 71(4), 959-971.

Brossier, R., Operto, S. \& Virieux, J., 2009. Seismic imaging of complex onshore structures by $2 \mathrm{D}$ elastic frequency-domain full-waveform inversion, Geophysics, 74(6), WCC105-WCC118.

Choi, Y., Min, D.-J. \& Shin, C., 2008. Two-dimensional waveform inversion of multi-component data in acoustic-elastic coupled media, Geophys. Prospect., 56(19), 863-881.

Clochard, V., Delépine, N., Labat, K. \& Ricarte, P., 2010. $\mathrm{CO}_{2}$ plume imaging using 3D pre-stack stratigraphic inversion: a case study on the Sleipner field, First Break, 28, 57-62.

De Barros, L. \& Dietrich, M., 2007. Full waveform inversion of shot gathers in terms of poro-elastic parameters, in Proceedings of the 69th EAGE Conference \& Exhibition, London, Extended Abstracts, no. P277.

De Barros, L. \& Dietrich, M., 2008. Perturbations of the seismic reflectivity of a fluid-saturated depth-dependent poroelastic medium, J. acoust. Soc. Am., 123(3), 1409-1420.

Domenico, S., 1984. Rock lithology and porosity determination from shear and compressional wave velocity, Geophysics, 49(8), 1188-1195.

Garambois, S. \& Dietrich, M., 2002. Full waveform numerical simulations of seismo-electromagnetic wave conversions in fluid-saturated stratified porous media, J. geophys. Res., 107(B7), 2148-2165.

Gassmann, F., 1951. Über die Elastizität poröser Medien, Vierteljahrsschrift der Naturforschenden Gesellschaft in Zürich, 96, 1-23.

Gauthier, O., Virieux, J. \& Tarantola, A., 1986. Two dimensionnal nonlinear inversion of seismic waveforms: numerical results, Geophysics, 51, 1387-1403.

Gélis, C., Virieux, J. \& Grandjean, G., 2007. Two-dimensional elastic full waveform inversion using Born and Rytov formulations in the frequency domain, Geophys. J. Int., 168(2), 605-633.

Gouveia, W.P. \& Scales, J.A., 1998. Bayesian seismic waveform inversion: parameter estimation and uncertainty analysis, J. geophys. Res., 103, 2759-2780.

Haartsen, M. \& Pride, S., 1997. Electroseismic waves from point sources in layered media, J. geophys. Res., 102(B11), 745-769.

Hicks, G. \& Pratt, R., 2001. Reflection waveform inversion using local descent methods: estimating attenuation and velocity over a gas-sand deposit, Geophysics, 66, 598-612.

Johnson, D., Plona, T. \& Kojima, H., 1994. Probing porous media with first and second sound. I. Dynamic permeability, J. appl. Phys., 76(1), 104-125.

Kennett, B., 1983. Seismic Wave Propagation in Stratified Media, Cambridge University Press, Cambridge, 342 pp.

Kormendi, F. \& Dietrich, M., 1991. Nonlinear waveform inversion of planewave seismograms in stratified elastic media, Geophysics, 56(5), 664-674.

Korringa, J., Brown, R., Thompson, D. \& Runge, R., 1979. Self-consistent imbedding and the ellipsoidal model for porous rocks, J. geophys. Res., 84, 5591-5598.

Lailly, P., 1983. The seismic inverse problem as a sequence of before stack migrations, in Proceedings of the Conference on Inverse Scattering: Theory and Applications, pp. 206-220, SIAM, Tulsa, OK.

Larsen, A.L., Ulvmoen, M., Omre, H. \& Buland, A., 2006. Bayesian lithology/fluid prediction and simulation on the basis of a Markov-chain prior model, Geophysics, 71(5), R69-R78.

Mavko, G., Mukerji, T. \& Dvorkin, J., 1998. The Rocks Physics Handbooks, Tools for Seismic Analysis in Porous Media, Cambridge University Press, Cambridge.

Mora, P., 1987. Nonlinear two-dimensional elastic inversion of multioffset seismic data, Geophysics, 52, 1211-1228.

Mora, M., Lesage, P., Valette, B., Alvarado, G., Leandro, C., Métaxian, J.-P. \& Dorel, J., 2006. Shallow velocity structure and seismic site effects at Arenal volcano, Costa Rica, J. Volc. Geotherm. Res., 152(1-2), 121-139. 
Morency, C., Luo, Y. \& Tromp, J., 2009. Finite-frequency kernels for wave propagation in porous media based upon adjoint methods, Geophy. J. Int., 179(2), 1148-1168.

Mosegaard, K. \& Tarantola, A., 2002. Probabilistic approach to inverse problems, International Handbook of Earthquake and Engineering Seismology, pp. 237-265, Academic Press Inc., London.

Mukerji, T., Jorstad, A., Avseth, P., Mavko, G. \& Granli, J.R., 2001. Mapping lithofacies and pore-fluid probabilities in a North Sea reservoir: seismic inversions and statistical rock physics, Geophysics, 66(4), 9881001.

O'Brien, G., 2010. 3D rotated and standard staggered finite-difference solutions to Biot's poroelastic wave equations: stability condition and dispersion analysis, Geophysics, 75(4), doi:10.1190/1.3432759.

Operto, S., Virieux, J., Dessa, J.X. \& Pascal, G., 2006. Crustal-scale seismic imaging from multifold ocean bottom seismometer data by frequencydomain full-waveform tomography: application to the eastern Nankai trough, J. geophys. Res., 111, doi:10.1029/2005JB003835.

Philippacopoulos, A., 1997. Buried point source in a poroelastic half-space, J. Eng. Mech., 123(8), 860-869.

Philippacopoulos, A., 1998. Spectral Green's dyadic for point sources in poroelastic media, J. Eng. Mech., 124(1), 24-31.

Pratt, R. \& Shipp, R., 1999. Seismic waveform inversion in the frequency domain, part II: fault delineation in sediments using crosshole data, Geophysics, 64(3), 902-914.

Pratt, R., Shin, C. \& Hicks, G., 1998. Gauss-Newton and full Newton methods in frequency-space seismic waveform inversion, Geophys. J. Int., 133(22), 341-362.
Pride, S., 2005. Relationships between seismic and hydrological properties, in Hydrogeophysics, pp. 253-284, Water Science and Technology Library, Springer, The Netherlands.

Pride, S., Gangi, A. \& Morgan, F., 1992. Deriving the equations of motion for porous isotropic media, J. acoust. Soc. Am., 92(6), 3278-3290.

Pride, S., Tromeur, E. \& Berryman, J., 2002. Biot slow-wave effects in stratified rock, Geophysics, 67, 271-281.

Pride, S. et al., 2003. Permeability dependence of seismic amplitudes, The Leading Edge, 22, 518-525.

Pride, S., Berryman, J. \& Harris, J., 2004. Seismic attenuation due to waveinduced flow, J. geophys. Res., 109, B01201.

Sahay, P., 2008. On the Biot slow S-wave, Geophysics, 73(4), 19-33.

Sirgue, L., Etgen, J. \& Albertin, U., 2008. 3D frequency domain waveform inversion using time domain finite difference methods, in Proceedings of the 70th EAGE Conference \& Exhibition, Rome, Extended Abstracts, no. F022.

Spikes, K., Mukerji, T., Dvorkin, J. \& Mavko, G., 2007. Probabilistic seismic inversion based on rock physics models, Geophysics, 72(5), 87-97.

Tarantola, A., 1984. The seismic reflection inverse problem, in Inverse Problems of Acoustic and Elastic Waves, pp. 104-181, SIAM, Philadelphia.

Tarantola, A., 1987. Inverse Problem Theory: Methods for data fitting and model parameter estimation, Elsevier, Amsterdam.

Tarantola, A. \& Valette, B., 1982. Generalized nonlinear inverse problems solved using the least squares criterion, Rev. Geophys. Space Phys., 20(2), 219-232.

Walton, K., 1987. The effective elastic moduli of a random packing of spheres, J. Mech. Phys. solids, 35, 213-226. 